\title{
In-situ Visualization and Analysis of Single Atom Dynamics in Chemical Reactions using Novel Environmental-Scanning Transmission Electron Microscopy (ESTEM)
}

\author{
Edward D. Boyes ${ }^{1}$, Thomas E. Martin ${ }^{2}$, David C. Lloyd ${ }^{2}$, Alec P. La Grow ${ }^{2}$, Robert W. Mitchell ${ }^{2}$, \\ Leonardo Lari ${ }^{2}$, Michael R. Ward ${ }^{2}$ and Pratibha L. Gai ${ }^{3}$ \\ 1. The York Nanocentre, Departments of Physics and Electronics, University of York, York,U.K. \\ 2. The York Nanocentre, Department of Physics, University of York, York, U.K. \\ 3. The York Nanocentre, Departments of Chemistry and Physics, University of York, York, U.K.
}

Heterogeneous gas-solid catalyst reactions take place at the atomic level, and understanding and controlling complex catalytic reactions at the atom level are crucial for the development of improved processes and materials. To visualize and analyse gas-catalyst reactions, we designed and constructed the first atomic resolution environmental transmission electron microscope (ETEM) [1], which has been exploited for commercial production and is used globally [2]. We have now developed the atomic resolution-ETEM further to support full ES(scanning)TEM functionality with aberration correction (AC), (AC ESTEM). Differentially pumped column sections are separated by re-positioned fixed beamline apertures. Single atom resolved high angle annular dark field (HAADF) imaging [Figure 1] and full analytical functionalities, including electron diffraction and EDX, are enabled for the first time under controlled chemical reaction conditions. They are maintained with high temperatures in a continuously flowing gas atmosphere around supported nanoparticle catalysts while retaining single atom sensitivity for atom-by-atom analysis of critical catalytic processes [3-5].

The AC ESTEM instrument in our laboratory has been used to obtain new insights into processes of metal nanoparticle catalyst deactivation [4], which has both economic and other societal importance; including for scientifically informed environmental management and redox reactions in nanoparticles [6]. Gas pressures used cover sample surfaces with many thousands of monolayers of gas per second and are generally fully adequate to flood the surface with gas molecules. They drive the chemistry under conditions defined in surface science [7)] as 'high pressure' and which minimise electron beam effects. Catalysis is both a massive industry and productive with highly leveraged investments in catalysis processes and plants. However in many applications it is still relatively inefficient compared to what might be possible with a better informed knowledge base generating strategies to restrain deactivation processes in emission control catalysis [8]. The AC ESTEM supports quantitative atom-by-atom analysis of the underlying mechanisms of sintering and catalyst deactivation through atom detachment, migration and reattachment at lower temperatures around constant particle centres of gravity $[9,10]$. This is compared with higher temperature modes of particle migration and coalescence with nanostructure attributes influencing local outcomes. Both have now been studied with new levels of single atom precision using the novel capabilities in our laboratory; leading to a better informed understanding of the technical options and enabling developments needed going forward [11].

References:

[1] E. D. Boyes and P. L. Gai, Ultramicroscopy 67 (1997), p. 219.

[2] P.L Gai et al, MRS Bulletin 32 (2007), p. 1044.

[3] P.L. Gai and E.D. Boyes, Micrsco. Res.Tech. 72 (2009), p. 153.

[4] E. D. Boyes et al, Ann Phys (Berlin) 525 (2013), p. 423. 
[5] P.L. Gai et al, Chem.Phys.Lett. 592 (2014), p. 355.

[6] A. P. La Grow et al, JACS 139 (2017), p. 179.

[7] G. Somorjai et al, PCCP 9 (2007), p. 3501.

[8] M. R. Ward et al, ChemCatChem 4 (2012), p. 1622.

[9] T. E. Martin, P.L. Gai and E. D. Boyes, ChemCatChem 7 (2015), p. 3705.

[10] E. D. Boyes and P. L. Gai, MRS Bulletin 40 (2015), p. 600.

[11] A grant from the EPSRC (UK) supporting this research is acknowledged. PLG and EDB thank the EPSRC (UK) for the research grant EP/ J0118058/1; for PhD studentships

for TEM, DCL and PDRAs for APL, RWM and MRW. (*Emails: pratibha.gai@york.ac.uk and ed.boyes@york.ac.uk)

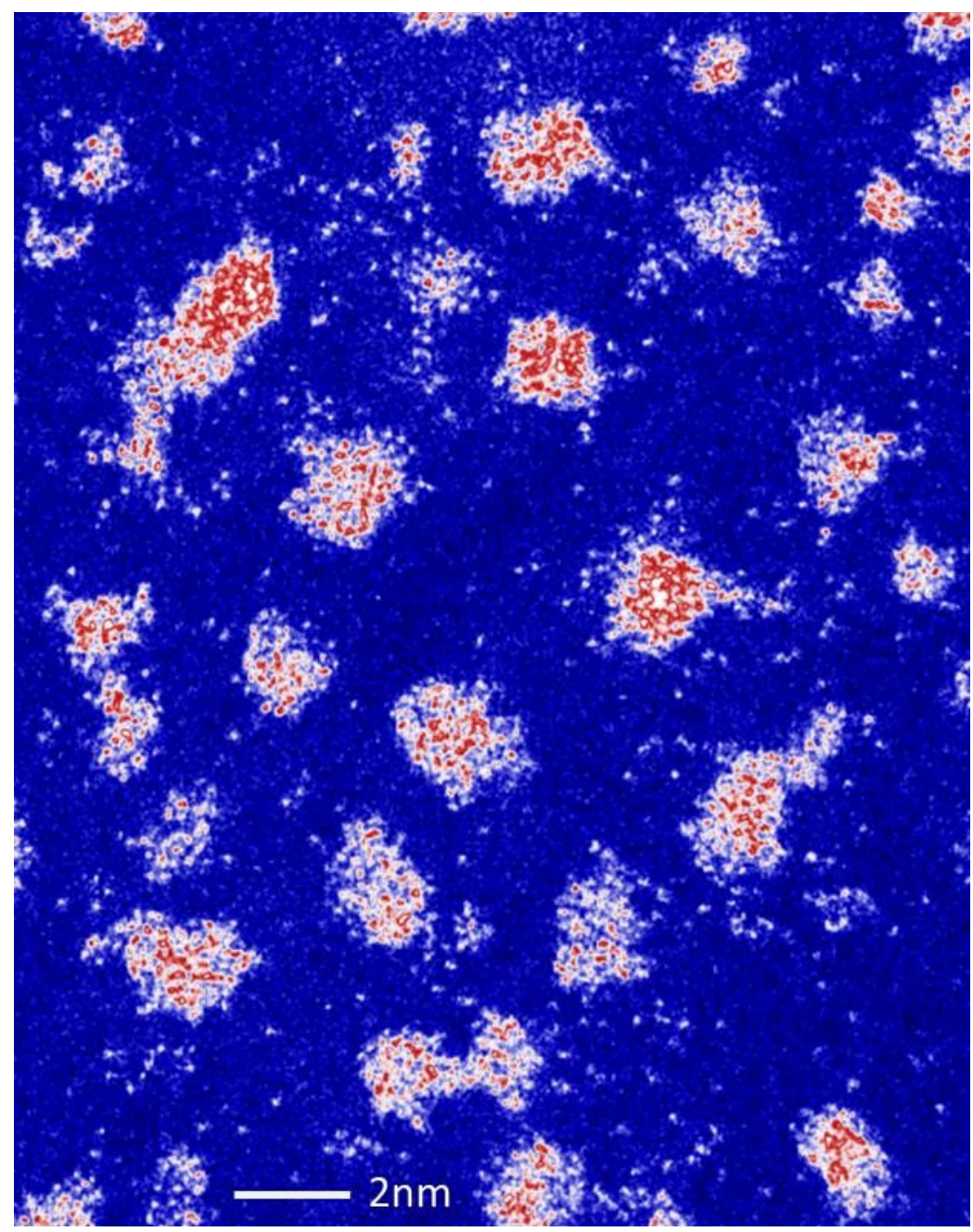

Figure 1. Single atoms and clusters of Pt on carbon in hydrogen gas 